\begin{tabular}{|c|l|}
\hline Title & Examination of Radio Frequency Blackout for an Inflatable V ehicle During A tmospheric Reentry \\
\hline Author(s) & Takahashi, Y usuke; Y amada, Kazuhiko; A be, Takashi \\
\hline Citation & $\begin{array}{c}\text { Journal of Spacecraft and Rockets, 51(2), 430-441 } \\
\text { https://doi.org/40.2514/1.A 32539 }\end{array}$ \\
\hline Issue Date & 201403 \\
\hline Doc URL & http://hdl.handle.net/2115/56572 \\
\hline Rights & ○ 2014 A merican Institute of A eronautics and A stronautics \\
\hline Type & article (author version) \\
\hline File Information & Examination of Radio Frequency Blackout for an Inflatable V ehicle during A tmospheric Reentry.pdf \\
\hline
\end{tabular}

Instructions for use 


\title{
Examination of Radio Frequency Blackout for an Inflatable Vehicle during Atmospheric Reentry
}

\author{
Yusuke Takahashi ${ }^{1}$ \\ Hokkaido University, Kita 13 Nishi 8, Kita-ku, Sapporo, Hokkaido, 060-8628, Japan, \\ Kazuhiko Yamada ${ }^{2}$ and Takashi $\mathrm{Abe}^{3}$, \\ Japan Aerospace Exploration Agency, 3-1-1 Yoshinodai Chuo-ku, Sagamihara, Kanagawa \\ 252-5210, Japan
}

\begin{abstract}
Numerical simulations of the plasma flow and electromagnetic wave around a membraneaeroshell type reentry vehicle were performed using various physical model combinations, and the possibility of radio frequency blackout of transceiver antenna embedded at the rear of the vehicle was investigated. The flow field was assumed to be in thermochemical nonequilibrium, and it was described by the Navier-Stokes equations with a multitemperature model and the equation of state. The simulations were performed for several altitudes including the highest heat flux point according to reentry orbit data. Through these computations, the detailed distributions of the flow-field properties in the shock layer and wake region were successfully obtained. To evaluate possibility of the radio frequency blackout during atmospheric reentry, the distribution of the electron number density around the inflatable vehicle was clarified. A frequency-dependent finite-difference time-domain method was used for simulations of electromagnetic waves, and the physical properties were obtained from the computational results of the plasma flow calculation. Electromagnetic wave behaviors in an ionized gas region behind the inflatable vehicle were investigated. It was found that the number density of electrons was sufficiently small and that the electromagnetic waves can propagate with less reflection and no attenuation. These results suggest that radio frequency blackout may not occur during the atmospheric reentry of the inflatable vehicle.
\end{abstract}

\section{Nomenclature}

$\mathrm{B}=$ magnetic flux density vector, $\mathrm{T}$

$D=$ effective diffusion coefficient, $\mathrm{m}^{2} / \mathrm{sec}$

$\mathrm{D}=$ electric flux density vector, $\mathrm{C} / \mathrm{m}^{2}$

$e=$ electric charge, $\mathrm{C}$

$\mathbf{E}=$ electric field vector, $\mathrm{V} / \mathrm{m}$

$f=$ frequency, $\mathrm{Hz}$

$\mathbf{J}=$ current density vector, $\mathrm{A} / \mathrm{m}^{2}$

$\mathbf{H}=$ magnetic field vector, $\mathrm{A} / \mathrm{m}$

$m=$ mass, $\mathrm{kg}$

$n=$ number density, $1 / \mathrm{m}^{3}$

$t=$ time, sec

$T=$ temperature, $\mathrm{K}$

$\varepsilon_{\mathrm{r}}=$ relative permittivity

$\varepsilon_{0}=$ permittivity in free space, $\mathrm{N} / \mathrm{V}^{2}$

\footnotetext{
${ }^{1}$ Assistant Professor, Faculty of Engineering; ytakahashi@eng.hokudai.ac.jp.

${ }^{2}$ Assistant Professor, Institute of Space and Astronautical Science.

${ }^{3}$ Professor, Institute of Space and Astronautical Science.
} 


$$
\begin{array}{lll}
\mu_{0} & = & \text { permeability in free space, } \mathrm{N} / \mathrm{A}^{2} \\
\nu_{\mathrm{c}} & =\text { collision frequency, } \mathrm{Hz} \\
\sigma & =\text { conductivity, } \mathrm{S} / \mathrm{m} \\
\chi & =\text { electric susceptibility } \\
\omega_{\mathrm{p}} & = & \text { plasma angular frequency, } \mathrm{rad} / \mathrm{sec}
\end{array}
$$

\begin{tabular}{|c|c|c|}
\hline \multicolumn{3}{|c|}{ Subscripts } \\
\hline e & $=$ & electron \\
\hline ex & $=$ & electronic excitation \\
\hline $\mathrm{p}$ & $=$ & plasma \\
\hline rot & $=$ & rotation \\
\hline$s$ & $=$ & species \\
\hline $\operatorname{tr}$ & $=$ & translation \\
\hline$v$ & $=$ & viscous \\
\hline vib & $=$ & vibration \\
\hline
\end{tabular}

\section{Introduction}

Membrane-aeroshell (inflatable) reentry vehicles are a candidate of future space transport system. Inflatable vehicles, which were firstly proposed in the $1960 \mathrm{~s}$, afford remarkable advantages, such as lower-ballistic-coefficient flight during atmospheric reentry. In the years, since the National Aeronautics and Space Administration (NASA), European Space Agency (ESA), and Japan Aerospace Exploration Agency (JAXA) have conducted several studies and demonstration flight of such vehicles. In general, inflatable vehicle can be decelerated at higher altitudes than conventional capsules. This can afford some advantages in the entry, descent, and landing (EDL) approach, such as lower heat load due to aerodynamic heating and soft landing without requiring a parachute system.

One of the most important issues in the EDL approach is the radio frequency $(\mathrm{RF})$ blackout, that is, the cutoff of communications between the reentry vehicle and the ground stations and/or data-relay satellites. During atmospheric reentry from a low Earth orbit, the vehicle moves at $\sim 7 \mathrm{~km} / \mathrm{s}$, and a strong shock-wave is formed at its front. Air is dissociated and ionized by strong aerodynamic heating in the shock-wave layer. A large number of electrons and ions are generated, and these charged particles cover the vehicle surface. RF blackout may be due to the plasma layer on the vehicle surface attenuating or reflecting the electromagnetic waves used for telecommunication, navigation, guidance, and control.

RF blackout has been observed in many reentry flight tests such as RAM-C II, OREX, and ARD. These vehicles had a relatively high ballistic coefficient, and therefore, they underwent severe aerodynamic heating at low altitudes where the density of air becomes high. At these altitudes, the number densities of electrons and ions also inevitably increase. Now, an inflatable vehicle is not or is minimally affected by RF blackout, because it decelerates at a high altitude where the density of air is low, reducing the plasma density generated in the shock wave.

In recent years, JAXA has initiated the Membrane Aeroshell for Atmospheric-entry Capsule (MAAC) project toward the development of an inflatable reentry vehicle. Thus far, experimental and numerical research and development on the elemental technologies of a future inflatable vehicle has been performed. Free flight tests using a scientific balloon were 
performed in 2004 and 2009 [1]. Then, a reentry demonstration flight was successfully conducted out using a sounding rocket on August 8, $2012[2,3]$. One of the next important milestones in the MAAC project is called TITANS which involves an atmospheric reentry mission from a low Earth orbit. Because this mission also focuses on the demonstration of recovery, RF blackout is a significant concern from the viewpoint of precise determination of the vehicle position and control. Hence, for the safe design of an inflatable vehicle for atmospheric reentry, it is important to understand the RF blackout phenomenon.

This study focuses on the RF blackout phenomenon around an inflatable vehicle. Its objective is to clarify the possibility of RF blackout during the atmospheric reentry of TITANS. Because the RF blackout phenomenon is affected by the flow properties around the vehicle, specifically, the number density of electrons, we need to clarify the distribution of charged particles. However, it is difficult to accurately reproduce the environment in which $\mathrm{RF}$ blackout is caused by using ground testing facilities such as hypersonic wind tunnel, archeated wind tunnel, and ballistic range. Now, in recent times, remarkable progress has been achieved in the development of high-performance computers and algorithms for numerical simulation. Numerical prediction has become a powerful tool for investigating the details of phenomena such as a flow field or an electromagnetic wave. In the present study, we investigate plasma flows and RF blackout for TITANS using numerical simulation methods. In the last few decades, computational fluid dynamics (CFD) has been shown to be effective for flow field prediction. In addition, it is important to investigate the reflection and attenuation of electromagnetic waves around the vehicle in detail. For the simulation of electromagnetic waves in plasma, the finite-difference time-domain (FDTD) method or frequency-dependent FDTD (FD2TD) method are effective simulation tools [4]. In particular, because plasma is a frequency-dependent medium, the FD2TD method is more appropriated. Here, based on the computational results obtained by the simulation of plasma flow using CFD, the possibility of RF blackout is evaluated by the simulation of electromagnetic waves using FD2TD.

When decelerating at a high altitude, the flow field around a vehicle tends to be in a thermochemical nonequilibrium state. It is necessary to construct the nonequilibrium flow model accurately in oder to predict the distribution of the number density of electrons. To model the thermal nonequilibrium more accurately, the present flow field is described by the Navier-Stokes (NS) equations using a multitemperature model that includes the translational $\left(T_{\text {tr }}\right)$, rotational $\left(T_{\text {rot }}\right)$, vibrational $\left(T_{\text {vib }}\right)$, and electron $\left(T_{\mathrm{e}}\right)$ temperatures. Thus far, many studies of plasma physics and nonequilibrium phenomena, and several thermochemical models of various aspects of a high-temperature gas, such as the chemical reaction rate, transport properties, and relaxation time of internal energy exchange, have been developed. However, because complete physical data for an internal-energy-exchange model are not yet available, we have to be carefully when using these models. In particular, models of relaxation between the electronic excitation and other energy modes involve some unclear points. Hence, the electron energy and electronic-excitation energy modes are assumed to be in equilibrium $\left(T_{\mathrm{ex}}=T_{\mathrm{e}}\right)$, and the latter energy is not added to the former in this model. Moreover, there currently exist no physical and numerical models to predict various high-enthalpy flows with high accuracy. This is because the physical model itself involves some uncertainty. To treat such issues, it is considered that a comparison of numerical results calculated using various physical model combinations is required. 


\section{Formulation of Flow Field}

\subsection{TITANS Reentry Vehicle}

Figure 1 shows a schematic view of the TITANS inflatable reentry vehicle. First, a capsule with a compactly packed aeroshell is transported to a distinct orbit (e.g., ISS orbit). As gas is injected into the torus tube, TITANS rapidly expands the membrane aeroshell under vacuum and zero-gravity conditions. After deployment, the flare-type membrane aeroshell, which also plays the roles of parachute and float in landing, is sustained by an inflatable torus. Then, the vehicle kicks into an atmospheric reentry orbit. Owing to its large area and light weight aeroshell, TITANS can achieve low-ballistic-coefficient flight during atmospheric reentry. Because aeroshell deployment is accomplished before deorbit, the inflatable vehicle is expected to fly using the EDL approach without any critical operations such as parachute extraction being required. TITANS mainly comprises three components -capsule, aeroshell, and inflatable torus- as shown in Fig. 2. The capsule is a spherical cone with a nose radius of $500 \mathrm{~mm}$ from the apex to the base and a base radius of $250 \mathrm{~mm}$. The membrane aeroshell has a flare angle of $70^{\circ}$ and frontal projected diameter of $2500 \mathrm{~mm}$, and it connects with the inflatable torus. The inflatable torus has a tube diameter of $150 \mathrm{~mm}$. During inflation, the overall length of TITANS becomes $2500 \mathrm{~mm}$ in diameter. TITANS is basically designed to fly with no angle of attack during atmospheric reentry. A transceiver antenna is installed in the aft part of the capsule, using which the vehicle communicates with the ground station via a data-relay satellite.

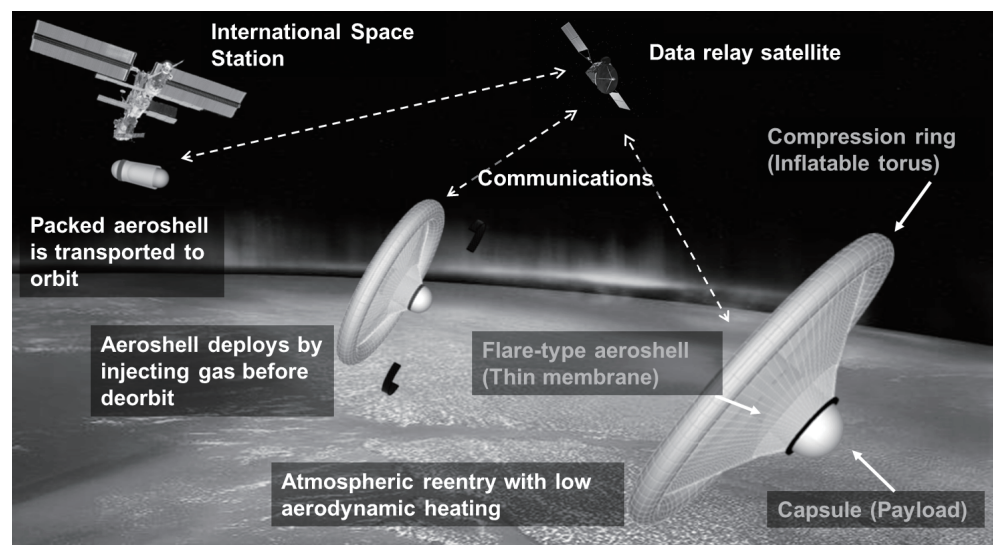

Figure 1: Schematic view of TITANS reentry vehicle

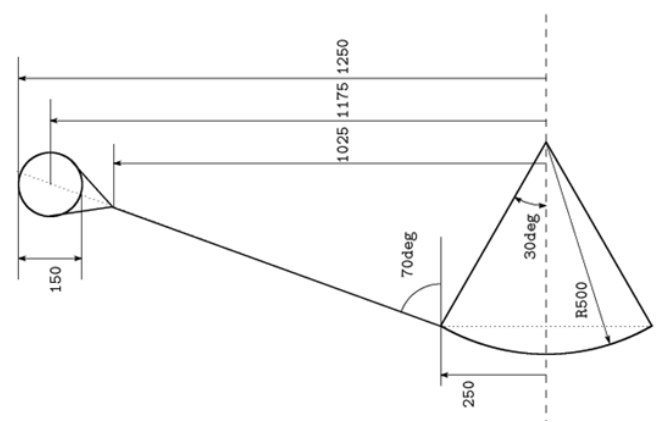

Figure 2: TITANS's configuration 


\section{$2.2 \quad$ Reentry Flight Trajectory}

Figure 3 shows the reentry trajectory profile of the altitude, reentry velocity, and heat flux versus elapsed time after deorbit for TITANS. The trajectory design is determined by solving the equation of motion for reentry orbit. It is planned that the capsule will first deorbit from an initial circular orbit of $400 \mathrm{~km}$ altitude with a ballistic coefficient of $2.0 \mathrm{~kg} / \mathrm{m}^{2}$. Reentry occurs with an entry velocity of $7588 \mathrm{~m} / \mathrm{s}$, and the deorbit delta velocity is assumed to be 80 $\mathrm{m} / \mathrm{s}$. The heat flux is evaluated by Tauber's model [5]. Owing to the low-ballistic-coefficient flight, the vehicle can be decelerated at a relatively high altitude. The heat flux gradually increases after deorbit and reaches a maximum of $126 \mathrm{~kW} / \mathrm{m}^{2}$ at an altitude of $89.3 \mathrm{~km}$. Then, as the reentry velocity and altitude rapidly decrease, aerodynamic heating is observed to reduce.

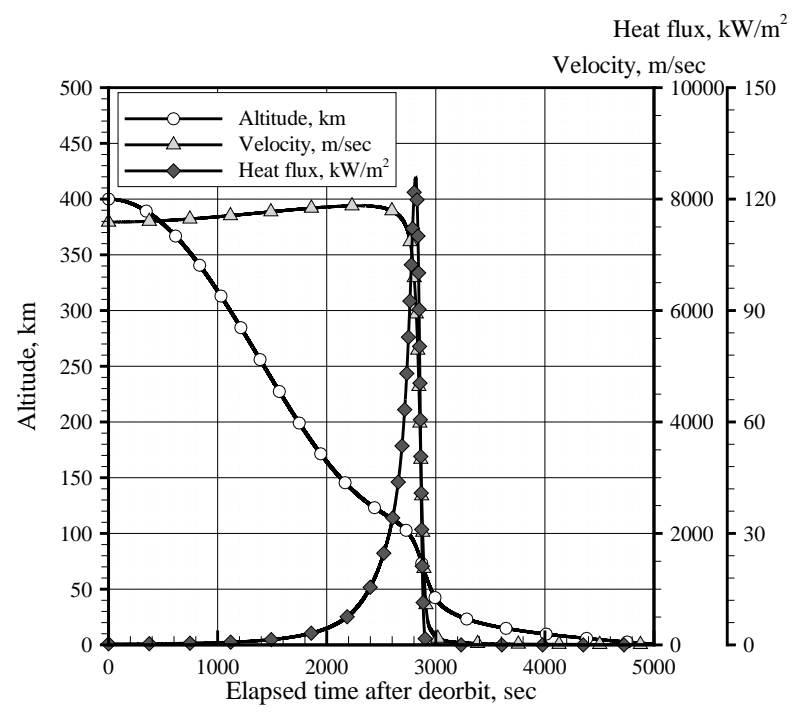

Figure 3: Trajectory profile of TITANS mission

\subsection{Governing Equations}

In this study, the following assumptions are employed: (1) The flow is laminar, steady, continuum and axisymmetric. (2) The inflow gas is air. (3) The flow field is in thermochemical nonequilibrium and the temperature is separated into translational $\left(T_{\mathrm{tr}}\right)$, rotational $\left(T_{\text {rot }}\right)$, vibrational $\left(T_{\text {vib }}\right)$, and electron $\left(T_{\mathrm{e}}\right)$ temperatures. Note that the electronic-excitation energy mode and the electron energy are assumed to be equilibrated (i.e., electronic-excitation temperature: $T_{\mathrm{ex}}=T_{\mathrm{e}}$ ).

The flow field is described by the Navier-Stokes equations with a multi-temperature model and the equation of state. The NS equations include total mass, momentum, total energy, species mass, rotation energy, vibration energy and electron energy conservations. The equation system can be written in vector form as follows:

$$
\frac{\partial \mathbf{Q}}{\partial t}+\frac{\partial \mathbf{F}}{\partial x_{j}}=\frac{\partial \mathbf{F}_{v}}{\partial x_{j}}+\mathbf{W}
$$

where $\mathbf{Q}$ is the conservative vector. Furthermore, $\mathbf{F}$ and $\mathbf{F}_{v}$ are the vectors of inviscid and viscous terms, respectively, and $\mathbf{W}$ denotes the vector of the source term. 


\subsection{Transport Properties}

Transport properties, such as the viscosity, thermal conductivity and binary diffusion coefficients for a mixture gas are evaluated by Yos's formula, which is based on the first ChapmanEnskog approximation. The collision cross sections are given by Gupta's works [6]. The diffusion coefficients are expressed by Curtiss and Hirschfelder's formula [7]. Ambipolar diffusion is assumed for charged species as $D_{s}^{a}=\left(1+T_{\mathrm{e}} / T_{\mathrm{tr}}\right) D_{s}$, where $D_{s}$ is the effective diffusion coefficient of the ionic species.

\subsection{Thermochemical Nonequilibrium}

For chemical reactions in high-temperature air, the test gas is assumed to consist of 11 chemical species $\left(\mathrm{N}_{2}, \mathrm{O}_{2}, \mathrm{NO}, \mathrm{N}_{2}^{+}, \mathrm{O}_{2}^{+}, \mathrm{NO}^{+}, \mathrm{N}, \mathrm{O}, \mathrm{N}^{+}, \mathrm{O}^{+}\right.$and $\left.\mathrm{e}^{-}\right)$and 49 reactions are assumed to occur. The chemical reaction rate is determined using an Arrhenius type form. The reaction rate coefficients are obtained from Park's work [8]. Moreover, the backward reaction rate is evaluated from the corresponding equilibrium constant. The equilibrium constants, being functions of only temperature, are calculated by the curve-fit formula given in Ref. [9].

We considered the energy transfer between each of the internal energy modes: translationrotation (T-R) [10], translation-vibration (T-V) [11, 12], translation-electron (T-e) [13-15], rotation-vibration (R-V) [10], rotation-electron (R-e) [16, 17], and vibration-electron (V-e) [18]. The energy losses/releases for vibrations, $Q_{\mathrm{D}}^{\text {vib }}$, and rotations, $Q_{\mathrm{D}}^{\text {rot }}$, associated with chemical reactions reveal the dissociation energies for heavy-particle-impact reactions. These can be obtained using a non-preferential dissociation model [19]. In addition, the electron energy loss/release due to electron-impact dissociation, $Q_{\mathrm{D}}^{\mathrm{e}}$, and ionization, $Q_{\mathrm{I}}^{\mathrm{e}}$, are also considered.

\section{Numerical Procedure of Plasma Flow Simulation}

\subsection{General}

The governing equations are transformed to the generalized coordinate system and solved using a finite volume approach. All the flow properties are set at the center of a control volume. The inviscid fluxes in the flow-field equations are evaluated using the AUSMDV scheme [20] and all the viscous terms are calculated using the second-order central difference method. The spatial accuracy is thus essentially of the second order.

Time integration is performed using an implicit time-marching method. The governing equation system is transformed into the delta form and the solution is updated at each time step. When modeling the thermochemical nonequilibrium flow, we need to introduce a robust and effective time-integration scheme to overcome the stiffness caused by the large timescale difference between the chemical reactions and the fluid motion that results in a severe restriction of the Courant-Friedrich-Lewy condition. Therefore, we employ the lower-upper symmetric Gauss-Seidel (LU-SGS) method coupled with the point-implicit method [21].

\subsection{Boundary Conditions}

At the inflow, the freestream parameters are given, following the orbit data shown in Fig. 3. At the outflow, all the flow properties are determined by the zeroth extrapolation because 
the flow is supersonic in most regions. The non-slip condition for the velocity and the noncatalytic condition for the mass concentration are imposed at the wall surfaces. No pressure gradient is assumed to be normal to the wall. The translational $\left(T_{\text {tr }}\right)$, rotational $\left(T_{\text {rot }}\right)$ and vibrational $\left(T_{\mathrm{vib}}\right)$ temperatures are fixed at the wall and are set to be $300 \mathrm{~K}$. The electron temperature $\left(T_{\mathrm{e}}\right)$ is assumed to be adiabatic at the wall. An axisymmetric condition is imposed along the symmetry axis.

\subsection{Calculation conditions}

The present calculations are performed for six cases among altitudes of $100.2 \mathrm{~km}$ and 75.4 $\mathrm{km}$, as shown in Table 1. Inflow parameters such as the freestream velocity, density and temperature are set according to these flight conditions. The degree of ionization at the inflow is always set to $10^{-10}$. The calculation condition at an altitude of $89.3 \mathrm{~km}$ is set to be the baseline case at which the heat flux reaches the highest value in orbit. The heat fluxes are calculated by Tauber's model [5]. The computational grid system for the baseline case is shown in Fig. 4, although different grid systems are used at each altitude. Note that the calculation geometry at the rear part of TITANS is simplified. A membrane aeroshell is generally deformed by the aerodynamic force during atmospheric flight [22]. However, for simplicity, membrane deformation is not considered in this model because it is expected to have a small influence on the prediction of the distribution of the number density of electrons.

Table 1: Flight conditions

\begin{tabular}{c|cccccc}
\hline \hline Elapsed time, $\mathrm{s}$ & 2750 & 2782 & 2814 & 2834 & 2845 & 2863 \\
\hline Altitude, $\mathrm{km}$ & 100.2 & 95.6 & 89.3 & 84.3 & 81.0 & 75.4 \\
Density, $\mathrm{kg} / \mathrm{m}^{3}$ & $5.93 \times 10^{-7}$ & $1.33 \times 10^{-6}$ & $3.76 \times 10^{-6}$ & $8.09 \times 10^{-6}$ & $1.35 \times 10^{-5}$ & $3.12 \times 10^{-5}$ \\
Temperature, K & 191.3 & 188.6 & 204.8 & 212.6 & 214.0 & 216.3 \\
Velocity, $\mathrm{m} / \mathrm{s}$ & 7356.9 & 7014.5 & 6229.6 & 5328.0 & 4594.7 & 3234.5 \\
Heat flux, $\mathrm{kW} / \mathrm{m}^{2}$ & 83.3 & 107.5 & 125.6 & 114.0 & 93.3 & 47.9 \\
\hline \hline
\end{tabular}

\section{Electromagnetic Wave}

To evaluate the possibility of radio communication between the TITANS vehicle and the data-relay satellite, it is important to clarify the behavior of electromagnetic waves in the plasma region at the rear of the vehicle. Maxwell's equations for describing the behavior of electromagnetic waves are expressed as follows:

$$
\begin{aligned}
& \nabla \times \mathbf{E}=-\frac{\partial \mathbf{B}}{\partial t}, \\
& \nabla \times \mathbf{H}=\frac{\partial \mathbf{D}}{\partial t}+\mathbf{J}, \\
& \nabla \cdot \mathbf{D}=\rho_{\mathrm{em}} \\
& \nabla \cdot \mathbf{B}=0
\end{aligned}
$$




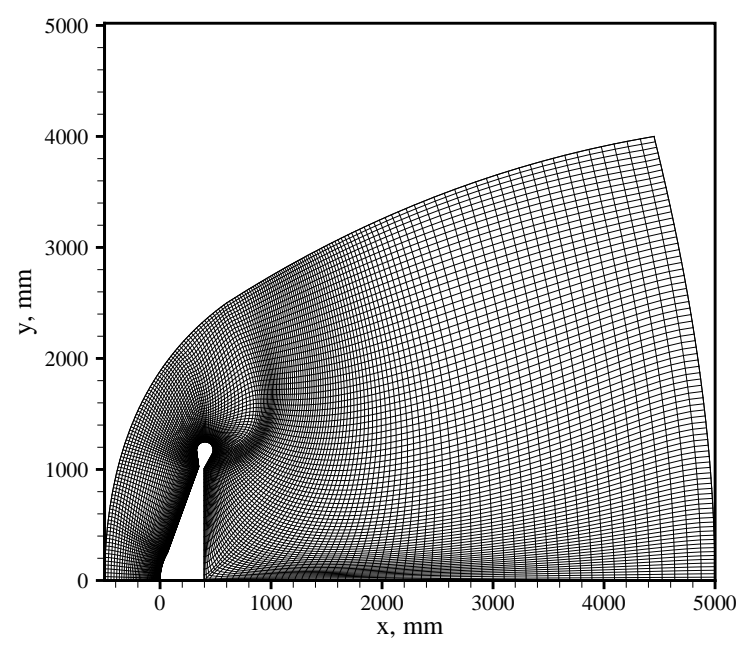

Figure 4: Computational grids of flow field

The FDTD method has been widely used for simulating electromagnetic wave propagation. However, in general, plasmas are frequency-dependent media whose dielectric constant and electrical conductivity vary over a frequency range. The FDTD formulation assumes that these media properties are independent of frequency, and this leads to the risk of obtaining incorrect results when applying frequency-dependent media. On the other hand, the FD2TD method is effective for the numerical simulation of Maxwell's equations in such frequencydependent media. In fact, Kinefuchi et al. [23] reported that FD2TD computational results of microwave propagation around a rocket plume showed good agreement with the experimental results. In addition, Cummer [24] proposed two effective FDTD methods for isotropic cold plasma. Generally, the time step of electromagnetic wave simulation in plasma is strictly limited and the Courant number should remain low because the speed of wave propagation becomes locally high with a decrease in the permittivity. However, in Cummer's study, even though the Courant number was set to one, fast and stable computations of the electromagnetic wave were successfully performed.

In the present FD2TD formulation, the complex relative permittivity $\left(\varepsilon_{\mathrm{r}}^{*}\right)$ assuming the first Drude dispersion is given by

$$
\varepsilon_{\mathrm{r}}^{*}=1+\frac{\omega_{\mathrm{p}}^{2}}{\omega\left(i \nu_{c}-\omega\right)}=1+\chi(\omega)
$$

where $\omega_{\mathrm{p}}, \nu_{\mathrm{c}}$, and $\chi(\omega)$ respectively represent the plasma angular frequency, electron collision frequency, and electric susceptibility at a given angular frequency $(\omega)$. Note that $i$ represents the imaginary unit. The complex relative permittivity can be expressed with the relative permittivity $\left(\varepsilon_{\mathrm{r}}\right)$ and dielectric conductivity $(\sigma)$ as follows:

$$
\varepsilon_{\mathrm{r}}^{*}=\varepsilon_{\mathrm{r}}+\frac{\sigma}{i \omega \varepsilon_{0}}
$$

where

$$
\begin{gathered}
\varepsilon_{\mathrm{r}}=1-\frac{\omega_{\mathrm{p}}^{2}}{\omega^{2}+\nu_{\mathrm{c}}^{2}}, \\
\sigma=\frac{\varepsilon_{0} \omega_{\mathrm{p}}^{2}}{\omega^{2}+\nu_{\mathrm{c}}^{2}} \nu_{\mathrm{c}} .
\end{gathered}
$$


Assuming that the electric field is constant during a time step $(\Delta t)$, the electric flux density at the $n$-th step is expressed as follows:

$$
\begin{aligned}
\mathbf{D}^{n} & =\varepsilon_{0} \mathbf{E}^{n}+\varepsilon_{0} \int_{0}^{n \Delta t} \chi(\tau) \mathbf{E}(n \Delta t-\tau) d \tau \\
& =\varepsilon_{0}\left(1+\chi^{0}\right) \mathbf{E}^{n}+\varepsilon_{0} \sum_{m=0}^{n-2} \mathbf{E}^{n-1-m} \chi^{m+1},
\end{aligned}
$$

where $\chi^{m}$ is defined using the electric susceptibility $\chi(\tau)$ in the time region as follows:

$$
\chi^{m}=\int_{m \Delta t}^{(m+1) \Delta t} \chi(\tau) d \tau
$$

Applying the Fourier transformation to $\chi(\omega)$ yields

$$
\chi^{m}=\frac{\omega_{\mathrm{p}}^{2}}{\nu_{\mathrm{c}}^{2}}\left\{\nu_{\mathrm{c}} \Delta t-\left(1-e^{-\nu_{\mathrm{c}} \Delta t}\right) e^{-\nu_{\mathrm{c}} m \Delta t}\right\} .
$$

Thus, the difference of the electric flux density in the time direction is given as follows:

$$
\mathbf{D}^{n}-\mathbf{D}^{n-1}=\varepsilon_{0}\left(1+\chi^{0}\right) \mathbf{E}^{n}-\varepsilon_{0} \mathbf{E}^{n-1}-\varepsilon_{0} \sum_{m=0}^{n-2} \mathbf{E}^{n-1-m} \Delta \chi^{m},
$$

where

$$
\Delta \chi^{m}=\chi^{m}-\chi^{m+1}=-\frac{\omega_{\mathrm{p}}^{2}}{\nu_{\mathrm{c}}^{2}}\left(1-e^{-\nu_{\mathrm{c}} \Delta t}\right)^{2} e^{-\nu_{\mathrm{c}} m \Delta t} .
$$

Equation (3) can be expressed using Eq. (13) as follows:

$$
\mathbf{E}^{n}=\frac{1}{1+\chi^{0}} \mathbf{E}^{n-1}+\frac{1}{1+\chi^{0}} \mathbf{\Phi}^{n-1}+\frac{\Delta t / \varepsilon_{0}}{1+\chi^{0}} \nabla \times \mathbf{H}^{n-\frac{1}{2}}
$$

and,

$$
\boldsymbol{\Phi}^{n-1}=\mathbf{E}^{n-1} \Delta \chi^{0}+e^{-\nu_{\mathrm{c}} \Delta t} \boldsymbol{\Phi}^{n-2}, n \geq 2 .
$$

As initial conditions, we set $\mathbf{E}^{0}=0, \mathbf{H}^{-\frac{1}{2}}=0$, and $\boldsymbol{\Phi}^{-1}=\boldsymbol{\Phi}^{0}=0$. In the present study, the magnetic permeability $(\mu)$ is assumed to not be dispersive. Hence, Eq. (2) is discretized as follows:

$$
\mathbf{H}^{n+\frac{1}{2}}=\mathbf{H}^{n-\frac{1}{2}}-\frac{\Delta t}{\mu} \nabla \times \mathbf{E}^{n} .
$$

The rotation terms in Eqs (15) and (17) are discretized by the central difference method in the spatial direction. The components of the electric field and magnetic field vectors are staggered on the Yee cell [25] as shown in Fig. 5. Therefore, the rotation of the electric fields in Eq. (17) is calculated with coplanar magnetic fields and that of the magnetic fields in Eq. (15) is calculated with coplanar electric fields.

The computational domain for electromagnetic waves is set to be a cuboid of $3 \mathrm{~m}$ length in the $x$ direction, $4 \mathrm{~m}$ width in the $y$ direction, and $4 \mathrm{~m}$ height in the $z$ direction. The computational grid nodes are set to 400 (x-direction), 200 (y-direction), and 200 (z-direction). TITANS is included in the cuboid, and the origin of the CFD computational domain corresponds to that of the electromagnetic wave calculation domain. The physical properties $\left(\omega_{\mathrm{p}}, \nu_{\mathrm{c}}\right)$ required in the present FD2TD are given by referring to the computational results 


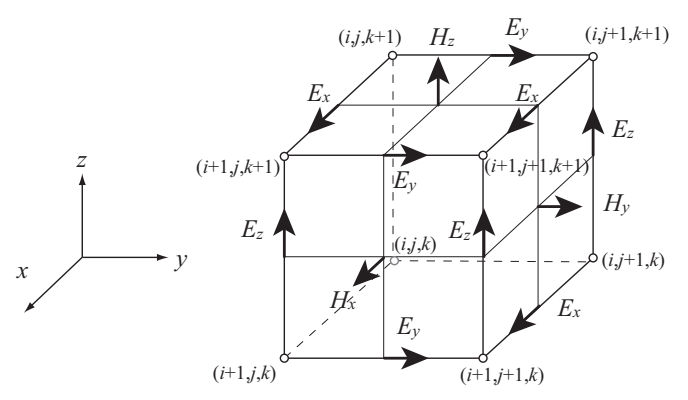

Figure 5: Yee cell.

obtained by CFD around TITANS. Note that the flow field computations are performed under the two-dimensional axisymmetric assumption and the flow properties are assumed to be uniform in the circumstance direction. A node in the FD2TD grid system is normally surrounded by eight grid points in the CFD domain. The plasma properties at the node positioned in the CFD cell are interpolated by the computed data on each point. On the other hand, the plasma parameters at a node in the FD2TD system that is positioned outside the CFD cell are obtained based on the parameters at the nearest boundary point in the CFD domain. The magnetic permeability $(\mu)$ is assumed to be the same as the free space $\left(\mu_{0}\right)$ for simplicity. Mur's absorption boundary condition [26] is imposed as a boundary condition at all six faces of the computational domain.

\section{$5 \quad$ Results and Discussion}

When the plasma above an antenna is assumed to be sufficiently thick, electromagnetic waves with frequency smaller than a given value are reflected by the plasma layer. The critical plasma frequency $f_{\mathrm{p}}$ in hertz is given as follows:

$$
f_{\mathrm{p}}=\frac{\omega_{\mathrm{p}}}{2 \pi}=\frac{1}{2 \pi} \sqrt{\frac{e^{2} n_{\mathrm{e}}}{\varepsilon_{0} m_{\mathrm{e}}}}
$$

where $e$ and $m_{\mathrm{e}}$ are the elementary charge and the mass of electron, respectively. Thus, the cut-off frequency preventing electromagnetic waves from reaching the antenna can be evaluated using the critical number density of electrons. If the link frequency between the vehicle and the ground station (and/or data-relay satellite) is set to be $1.6 \mathrm{GHz}$, the number density $\left(n_{\mathrm{e}, \text { crit }}\right)$ is found to be $3.167 \times 10^{16} 1 / \mathrm{m}^{3}$.

To evaluate RF blackout during atmospheric reentry, it is important to predict the distribution of the number density of electrons and the electromagnetic wave behavior around the vehicle. The transceiver antenna is embedded in the aft part of the TITANS capsule, as mentioned earlier. Hence, if the electron density in the wake region of TITANS is sufficiently small and attenuation and reflection of the electromagnetic waves are low, RF blackout will never occur during flight. In this section, first, we clarify the distributions of the flow-field properties for the baseline case. Then, we evaluate the electron number densities at several flight altitudes though plasma flow simulations using some physical models. Furthermore, we investigate the propagation of electromagnetic waves using the flow-field simulation results. Finally, we discuss RF blackout for TITANS. 


\subsection{Flow-field behavior for baseline case}

The present flow-field simulation model has been validated through a comparison with Tauber's model and experimental data obtained from JAXA's hypersonic wind tunnel. As shown in Fig. 6, the heat flux at the stagnation point of TITANS shows qualitatively good agreement with Tauber's model. However, the effective nose radius in Tauber's model does not correspond to the nose radius of TITANS, because the large aeroshell changes the feature of the shock layer compared to the traditional capsule configuration as is considered in Tauber's equation. On the other hand, the heat flux profiles of an inflatable vehicle (scaleddown TITANS configuration) calculated by CFD shows qualitatively good agreement with the experimental data, although this comparison is not shown here.

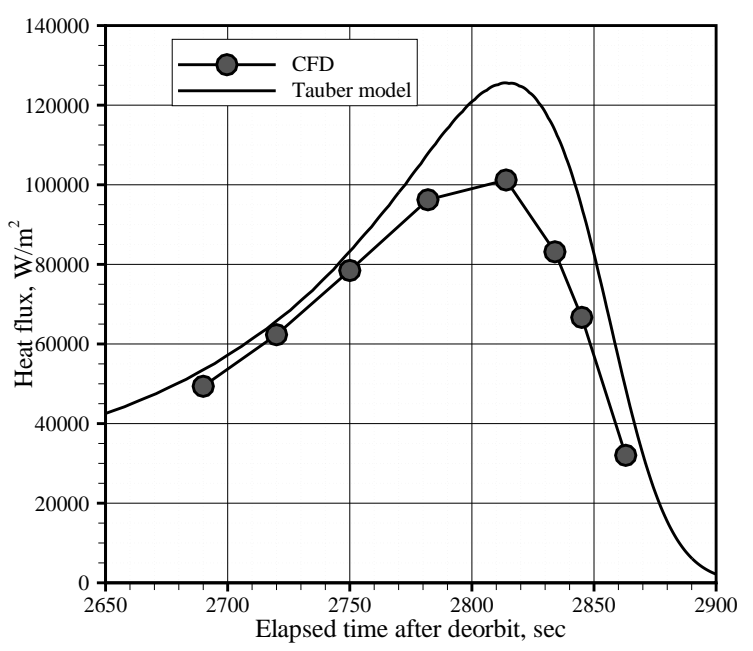

Figure 6: Comparison of heat flux histories at the stagnation point of TITANS between CFD and Tauber model.

Figures 7(a) and 7(b) respectively show axial profiles of temperatures and mole fractions along the center axis for the baseline case at an altitude of $89.3 \mathrm{~km}$ (elapsed time after deorbit: $2814 \mathrm{~s}$ ). In general, because the relaxation time between translational and rotational energy modes is short as that between vibrational and electron energy modes, the translational and rotational temperatures and vibrational and electron temperatures tend to be equilibrated, respectively. However, each of the temperatures is thoroughly separated, and strong thermal nonequilibrium appears in the flow field. The density of air is low at an altitude of $89.3 \mathrm{~km}$, and therefore, the number of collisions between particles inevitably also becomes low. This is why the flow field is close to being in nonequilibrium. At the same time, toward the surface from the free stream, the temperatures are seen to be equilibrated owing to an increase in the number of collisions with the increase in density.

As in the case of the temperature behavior mentioned above, chemical reactions appear to occur at a slow rate. The dominant reactions are dissociations of molecules, and the gas species in the shock layer mainly include molecular nitrogen, atomic nitrogen, and atomic oxygen. Moreover, a small number of electrons are also generated. This tendency near the center axis is similar near the inflatable torus, because the flare angle of the aeroshell is large and the shock layer is formed as a normal shock wave. In the wake region of TITANS, dissociation and ionization reactions rarely occur with decreases in temperature. Thus, to 
evaluate the behavior of charged particles in the vehicle's wake, it is important to understand the chemical reactions occurring in front of the vehicle.

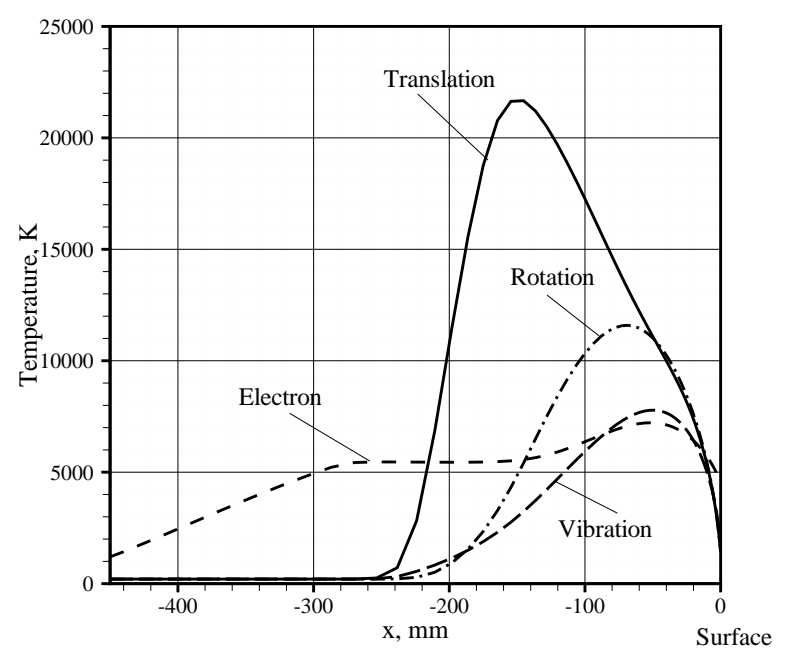

(a) Axial profile of temperatures

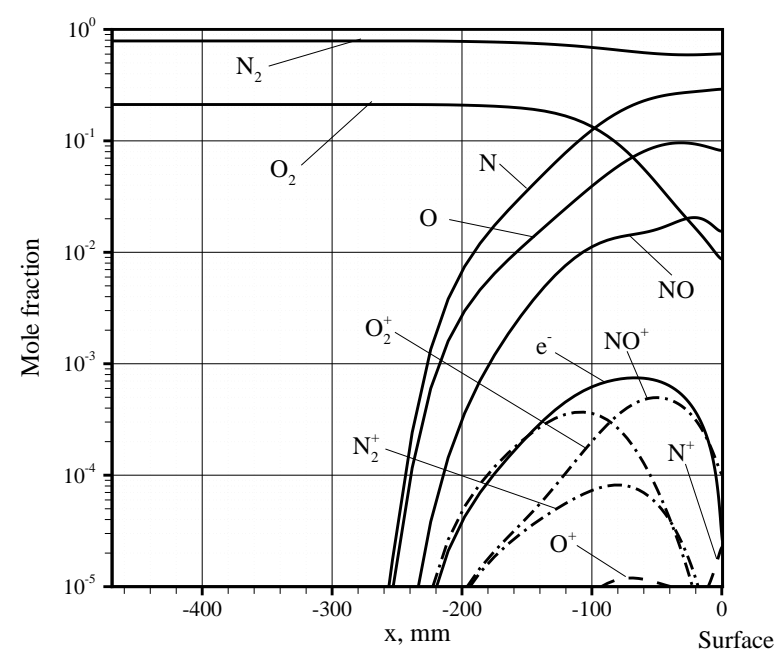

(b) Axial profile of mole fractions

Figure 7: Axial profiles of temperatures and mole fractions along the center axis in front of TITANS's flow field at an altitude of $89.3 \mathrm{~km}$ (elapsed time after deorbit: $2814 \mathrm{~s}$ )

Figure 8 shows the pressure distribution and stream traces for the baseline case at an altitude of $89.3 \mathrm{~km}$. It is confirmed that a shock wave is formed in front of the TITANS vehicle where the pressure is uniformly constant from the capsule to the torus. Compressed flow across the shock wave starts to expand near the inflatable torus. The flow appears to instantaneously separate above the top of the torus. This results in the formation of a large recirculation region behind TITANS's aeroshell. The separated flow is reattached at a position of $x \sim 2300 \mathrm{~mm}$ on the center axis. Thus, it is considered that electrons and ions generated in high-temperature gas hardly inflow into TITANS's wake region owing to the large recirculation, although there may exist particles drifting into the wake by a diffusion mechanism due to a gradient in the mass concentration.

\subsection{Electron density}

For the simulation of a high enthalpy flow, it is thought that the transport property, chemical reaction, and internal energy exchange models have large impacts on the prediction of the electron density distribution. This tendency is particularly noticeable at higher altitudes where the density is generally low and thermochemical nonequilibirum strongly appears. Unfortunately, no physical model combination is available for predicting the flow-field properties with great accuracy for various flight conditions. A physical model may show an inherent discrepancy with a true value. Hence, numerical results with a given model combination inevitably have some uncertainty. This reduces the reliability of numerical prediction. To try to solve this problem, we performed flow-field simulations using various model combinations and evaluated the uncertainty of RF blackout. The combinations used in the present calculation are summarized in Table 2.

The distributions of the number density of electrons around TITANS at each altitude are illustrated in Fig. 9. The black lines in these figures are drawn along isolines of the electron 


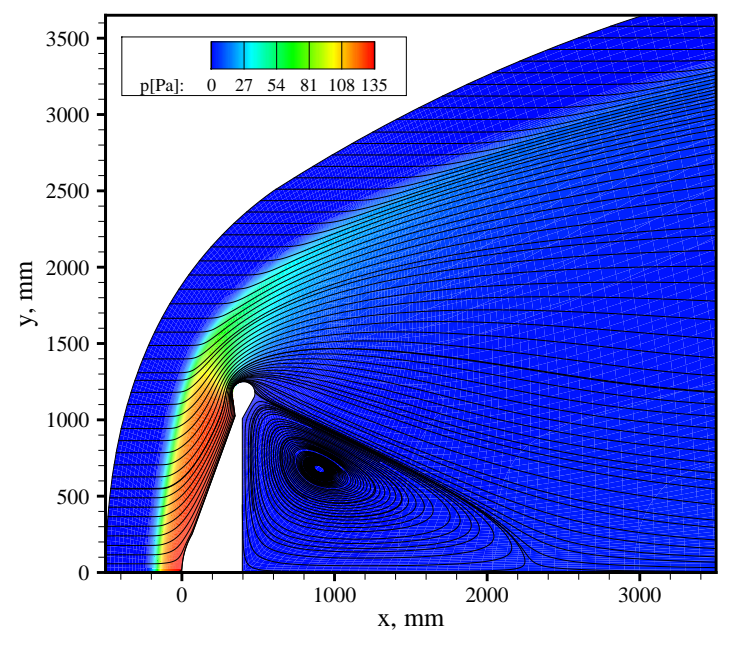

Figure 8: Pressure distribution and stream traces around TITANS at an altitude of $89.3 \mathrm{~km}$ (elapsed time after deorbit: $2814 \mathrm{~s}$ )

density values calculated using the 16 model cases, and the colored zones are drawn based on their averaged values. There appears a region in which the number density of electrons generated by the shock layer remains high from the top of the inflatable torus to TITANS's wake at each altitude. The ionized region is referred to as "plasma wake" in this study. Red-colored zones indicate regions where the electron density exceeds the critical value of $n_{\mathrm{e}, \mathrm{crit}}=3.167 \times 10^{16} 1 / \mathrm{m}^{3}$ for the link frequency of $1.6 \mathrm{GHz}$. When the electromagnetic waves pass through this region, attenuation, refraction, or reflection will occur. The redcolored zone is largest in area at an altitude of $89.3 \mathrm{~km}$ (Fig. 9(b)), and interestingly, it appears to show agreement with the heat flux values evaluated by Tauber's model (see also Table 1). It is clarified that the dispersion among the physical model combinations used here becomes large at high altitudes such an $95.6 \mathrm{~km}$, whereas it is small at lower altitudes. This is presumably related to the thermochemical nonequilibrium and the contribution of the viscous term in the NS equations. If the free stream density is sufficiently high and particle collisions are frequent, the flow field is close to thermochemical equilibrium. At this time, the internal energy exchange model and chemical reaction model have less influence on the prediction of flow properties. Moreover, when the Reynolds number increases with the density at lower altitudes, the contribution of the viscous term becomes relatively small compared with that of the convection term.

Many heat shields generally include impurities such as sodium and potassium. These impurities have low ionization levels and can increase the number density of electrons in the flow field around vehicles when being heated and ablated. Because RF blackout is sensitive to the presence of electrons, impurities can become a significant issue. On the other hand, TITANS was originally designed such that the surface temperature remains sufficiently low to avoid melting and outgassing, because of the relatively low maximum temperature of the fiber material that constitutes the membrane aeroshell and inflatable torus. Thus, we consider that few impurities are outgassed during TITANS's reentry flight.

At the rear of TITANS, because the number density of electrons decreases to well below the critical value, electromagnetic waves are expected to not be reflected at least. However, if the medium in which the electromagnetic wave propagates is sufficiently conductive, strong 
Table 2: Models combination

\begin{tabular}{ccccc}
\hline \hline case & Collision cross section & $T_{\text {tr }}, T_{\text {rot }}-T_{\text {vib }}$ & $T_{\text {tr }}-T_{\text {rot }}$ & Energy loss \\
\hline 1 & Capitelli [27]-Fertig [28] & $\mathrm{T}-\mathrm{V}(100 \%), \mathrm{R}-\mathrm{V}(0 \%)[11,12]$ & Parker [29] & Non-Preferential [19] \\
2 & Gupta [6] & $\mathrm{T}-\mathrm{V}(100 \%), \mathrm{R}-\mathrm{V}(0 \%)$ & Parker & Non-Preferential \\
3 & Capitelli-Fertig & $\mathrm{T}-\mathrm{V}(60 \%), \mathrm{R}-\mathrm{V}(40 \%)[10]$ & Parker & Non-Preferential \\
4 & Gupta & $\mathrm{T}-\mathrm{V}(60 \%), \mathrm{R}-\mathrm{V}(40 \%)$ & Parker & Non-Preferential \\
5 & Capitelli-Fertig & $\mathrm{T}-\mathrm{V}(100 \%), \mathrm{R}-\mathrm{V}(0 \%)$ & Park [10] & Non-Preferential \\
6 & Gupta & $\mathrm{T}-\mathrm{V}(100 \%), \mathrm{R}-\mathrm{V}(0 \%)$ & Park & Non-Preferential \\
7 & Capitelli-Fertig & $\mathrm{T}-\mathrm{V}(60 \%), \mathrm{R}-\mathrm{V}(40 \%)$ & Park & Non-Preferential \\
8 & Gupta & $\mathrm{T}-\mathrm{V}(60 \%), \mathrm{R}-\mathrm{V}(40 \%)$ & Park & Non-Preferential \\
9 & Capitelli-Fertig & $\mathrm{T}-\mathrm{V}(100 \%), \mathrm{R}-\mathrm{V}(0 \%)$ & Parker & Preferential [30] \\
10 & Gupta & $\mathrm{T}-\mathrm{V}(100 \%), \mathrm{R}-\mathrm{V}(0 \%)$ & Parker & Preferential \\
11 & Capitelli-Fertig & $\mathrm{T}-\mathrm{V}(60 \%), \mathrm{R}-\mathrm{V}(40 \%)$ & Parker & Preferential \\
12 & Gupta & $\mathrm{T}-\mathrm{V}(60 \%), \mathrm{R}-\mathrm{V}(40 \%)$ & Parker & Preferential \\
13 & Capitelli-Fertig & $\mathrm{T}-\mathrm{V}(100 \%), \mathrm{R}-\mathrm{V}(0 \%)$ & Park & Preferential \\
14 & Gupta & $\mathrm{T}-\mathrm{V}(100 \%), \mathrm{R}-\mathrm{V}(0 \%)$ & Park & Preferential \\
15 & Capitelli-Fertig & $\mathrm{T}-\mathrm{V}(60 \%), \mathrm{R}-\mathrm{V}(40 \%)$ & Park & Preferential \\
16 & Gupta & $\mathrm{T}-\mathrm{V}(60 \%), \mathrm{R}-\mathrm{V}(40 \%)$ & Park & Preferential \\
\hline \hline
\end{tabular}

attenuation occurs. It is possible that the waves can not pass through the ionized gas region behind TITANS. Thus, in order to judge the possibility of transit in plasma, electromagnetic wave simulations are considered necessary.

\subsection{Electromagnetic Wave Behavior}

The electromagnetic wave simulations are performed by the FD2DT method for each altitude. Plasma properties such as the permittivity $(\varepsilon)$, plasma angular frequency $\left(\omega_{\mathrm{p}}\right)$, and collision frequency $\left(\nu_{\mathrm{c}}\right)$ are obtained from the computational results of the present analysis model combinations. It is very complicated to analyze considering the detailed antenna configuration and antenna feeding methods. Thus, the generation of electromagnetic waves is simply modeled for a fundamental understanding in the present calculations. By inputting a sinusoidally electric field in the $z$ direction at the aft of the TITANS vehicle $(x=400 \mathrm{~mm}$, $-100 \leq y \leq 100 \mathrm{~mm}$ and $-100 \leq z \leq 100 \mathrm{~mm}$ ), transverse magnetic waves are generated as a wave source. Note that the amplitude of the electric field at the source is set to $1\left(E_{z 0}=1.0\right.$ $\mathrm{V} / \mathrm{m})$.

Figures 10(a) and 10(b) show the distributions of the permittivity and the dielectric conductivity (defined by Eqs. (8) and (9), respectively) in the $x-y$ plane $(z=0 \mathrm{~mm})$ of the FD2TD computational domain around TITANS. In the ionized gas, the permittivity is frequency-dependent and generally becomes low depending on the angular frequencies of the plasma $\left(\omega_{\mathrm{p}}\right)$ and the electromagnetic waves $(\omega)$ :

$$
\varepsilon(\omega)=\varepsilon_{0} \varepsilon_{\mathrm{r}}=\varepsilon_{0}\left(1-\frac{\omega_{\mathrm{p}}^{2}}{\omega^{2}+\nu_{\mathrm{c}}^{2}}\right)
$$

Thus, because the permittivity is negative in a region where $\omega_{\mathrm{p}}^{2}>\omega^{2}+\nu_{\mathrm{c}}^{2}$, the index of refraction becomes an imaginary number. Electromagnetic waves can not propagate into such 


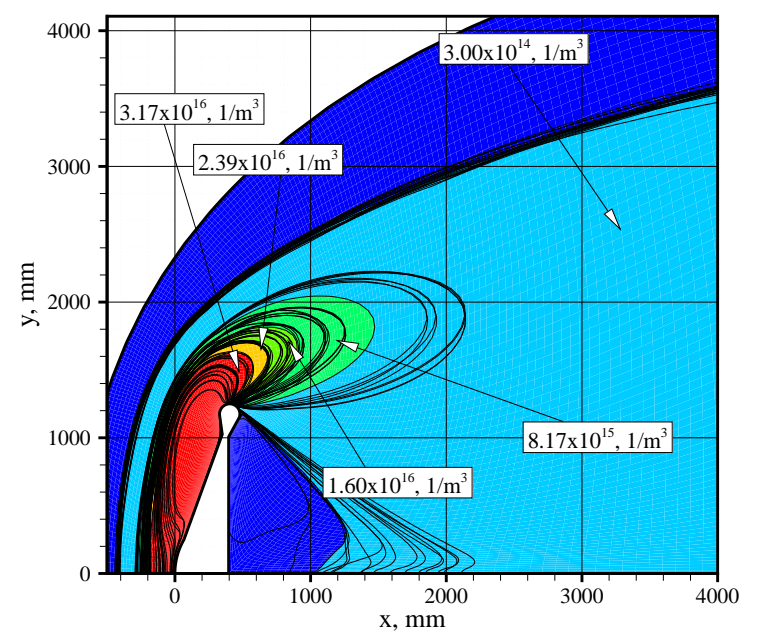

(a) altitude $95.6 \mathrm{~km}$

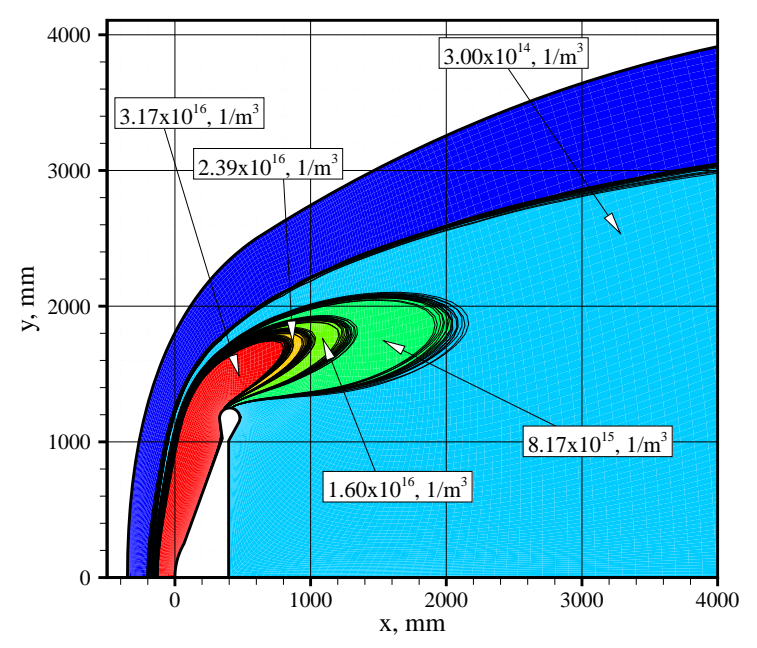

(c) altitude $84.3 \mathrm{~km}$

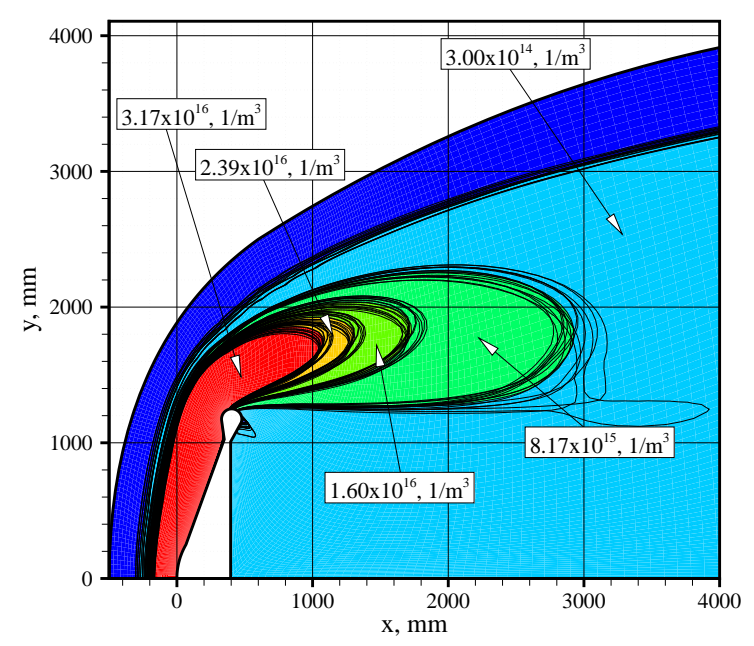

(b) altitude $89.3 \mathrm{~km}$

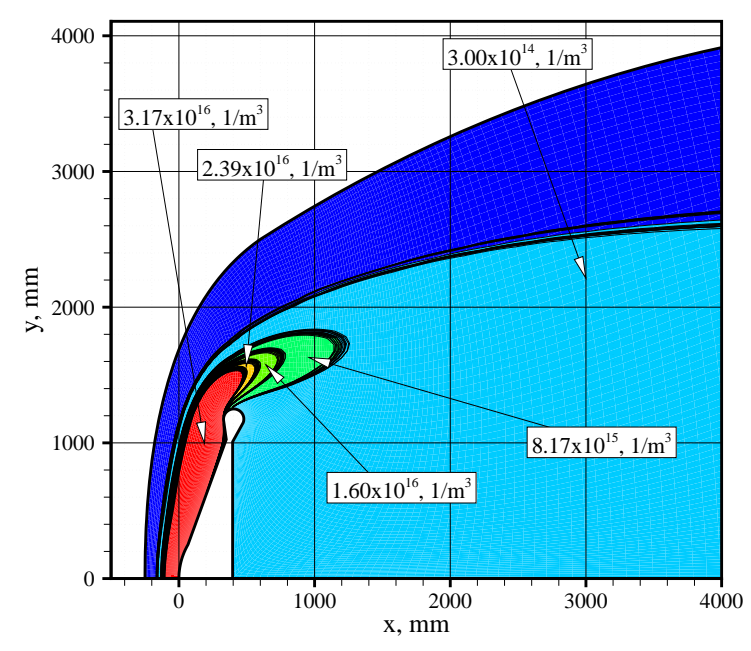

(d) altitude $80.0 \mathrm{~km}$

Figure 9: Distributions of number density of electrons around TITANS at each altitude.

a medium and are perfectly reflected on its surface. In addition, as the dielectric conductivity increases, the electromagnetic waves are strongly attenuated even if $\omega_{\mathrm{p}}^{2}<\omega^{2}+\nu_{\mathrm{c}}^{2}$. Because the number density becomes high and the plasma angular frequency $\left(\omega_{\mathrm{p}}\right)$ is larger than the link angular frequency $(\omega)$ in the shock layer in front of TITANS, the permittivity is negative. Then, in the plasma wake passing near the inflatable torus, the reflection of waves is not expected to occur because the permittivity recovers to a positive value, whereas the dielectric conductivity is sufficiently high. Electromagnetic waves are considered to hardly propagate in the medium. On the other hand, because the plasma angular frequency and dielectric conductivity are relatively low in the rarefied gas region behind TITANS, the permittivity value remains high. Thus, the electromagnetic waves are expected to propagate with no reflection and less attenuation.

Figures 11(a) and 11(b) respectively illustrate the magnitudes of the electric field and magnetic field of the electromagnetic waves around TITANS for model combination case 


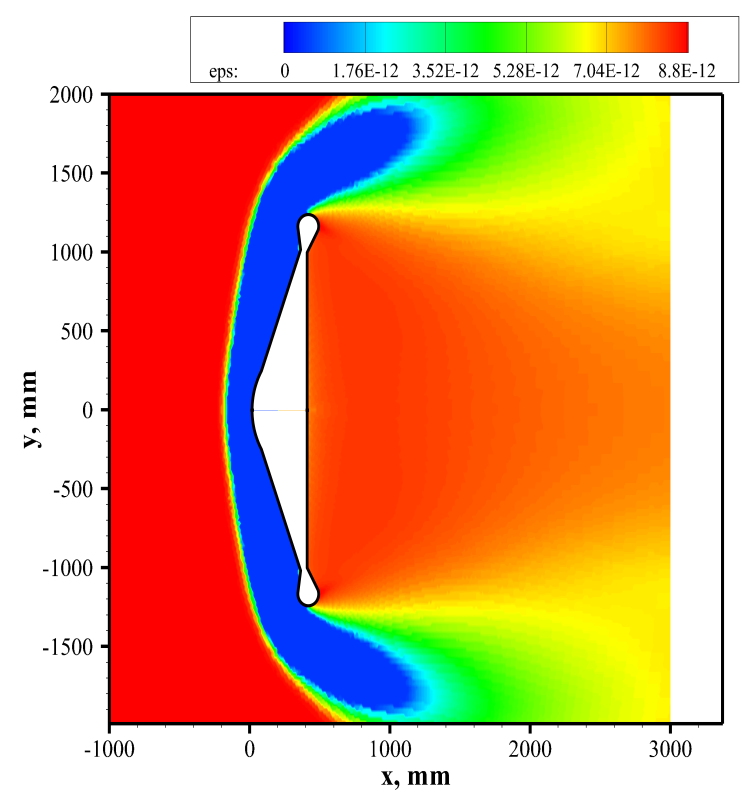

(a) Permittivity $\left(\mathrm{N} / \mathrm{V}^{2}\right)$

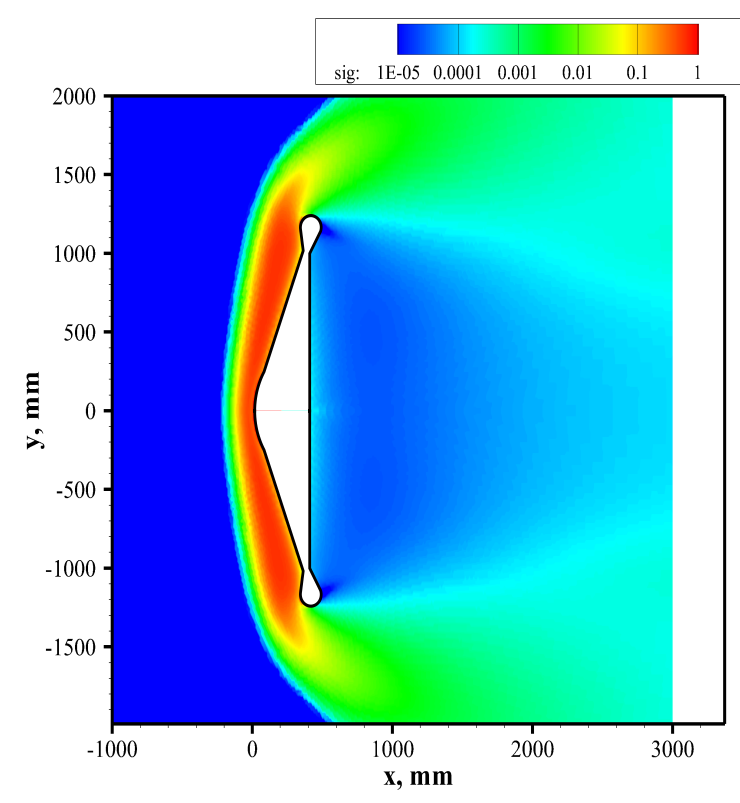

(b) Dielectric conductivity $(\mathrm{S} / \mathrm{m})$

Figure 10: Distributions of plasma angular frequency and permittivity in $x-y$ plane $(z=0$ $\mathrm{mm}$ ) of FD2TD computational domain around TITANS at an altitude of $89.3 \mathrm{~km}$.

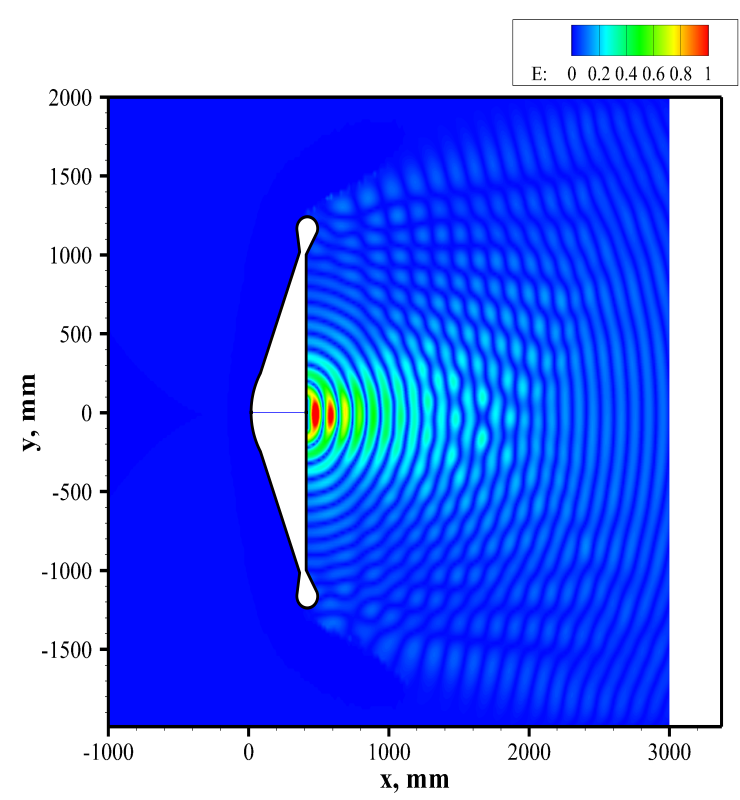

(a) Electric field magnitude $(\mathrm{V} / \mathrm{m})$

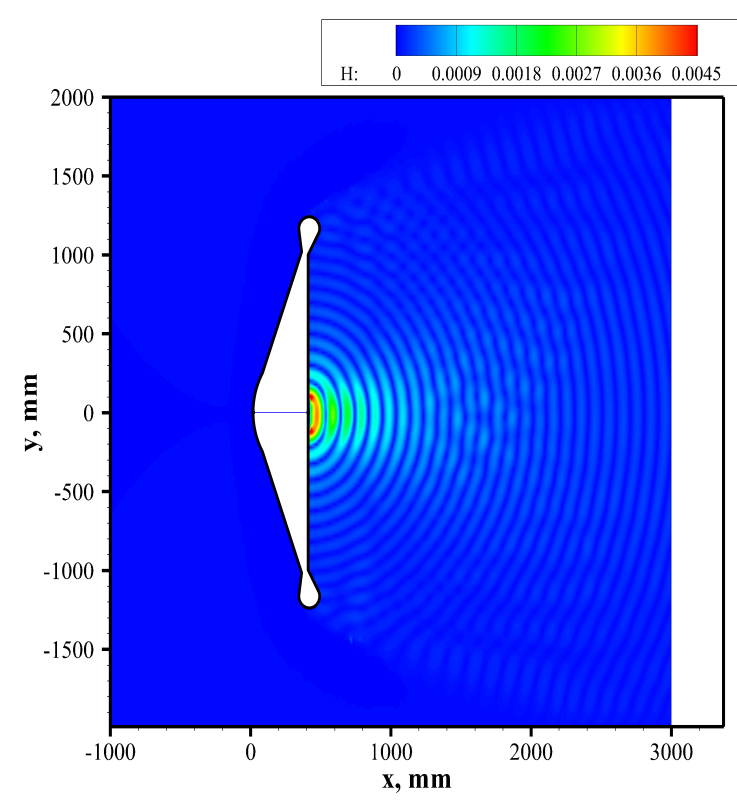

(b) Magnetic field magnitude $(\mathrm{A} / \mathrm{m})$

Figure 11: Distributions of electric field and magnetic field in $x-y$ plane $(z=0 \mathrm{~mm})$ of FD2TD computational domain around TITANS at an altitude of $89.3 \mathrm{~km}$.

1 at an altitude of $89 \mathrm{~km}$. Electromagnetic waves generated at the rear of TITANS are observed to propagate in the vehicle wake region and concentrically expand. On the surface of the plasma wake near the inflatable torus where the permittivity is less than zero and the dielectric conductivity is high, the waves are totally reflected or attenuated. It is indicated that the reflected waves interfere with waves propagating in a longitudinal direction in the rarefied gas region from $x=1000-2000 \mathrm{~mm}$ near the center line $(y=0 \mathrm{~mm})$. Because 
the plasma stretches from the shock layer near the torus, electromagnetic waves are hardly diffracted in front of the vehicle.

The electric field ( $z$-direction component, $E_{z}$ ) and magnetic field ( $y$-direction component, $\left.H_{y}\right)$ profiles along the center line $(y=0$ and $z=0 \mathrm{~mm})$ of the FD2TD computational domain around TITANS are shown in Figs. 12(a) and 12(b), respectively. For comparison, the electric and magnetic fields profiles computed assuming that the medium parameters, such as permittivity, around TITANS are the same as those in free space (i.e., $\varepsilon=\varepsilon_{0}$, $\omega_{\mathrm{p}}=0, \nu_{\mathrm{c}}=0$ and $\sigma=0$ ) are also shown in these figures ("free space assumption" in the figure legends). The electromagnetic wave is observed to be less attenuated by the ionized gas compared with the computational results with the free space assumption. On the other hand, it is confirmed that there appear phase leads by the plasma medium. This is because the speed of light in the plasma medium increases with the decrease in permittivity, according to $c=1 / \sqrt{\varepsilon \mu}$. Note that the decrease in a region of $x \sim 1700 \mathrm{~mm}$ is caused by interference of the reflected waves at the plasma wake near the inflatable torus.

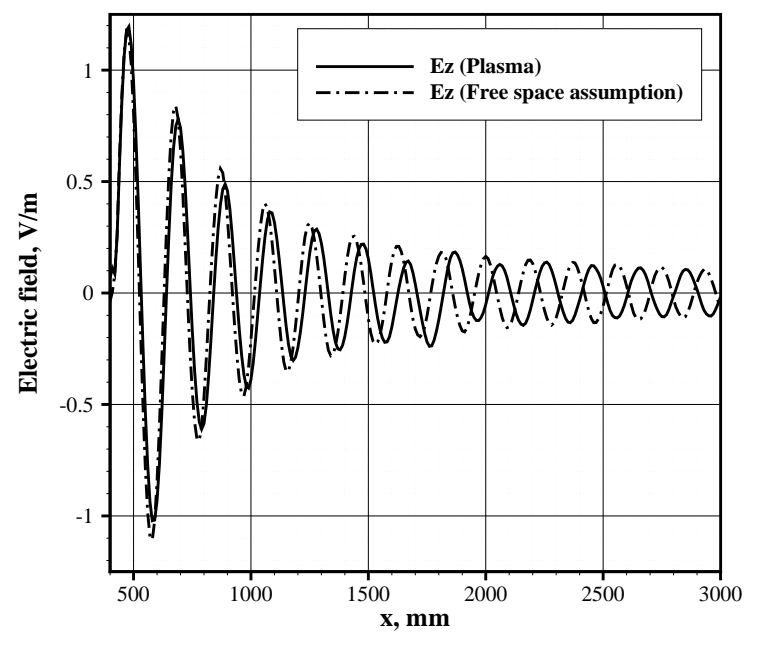

(a) $z$-direction component of electric field $\left(E_{z}\right)$

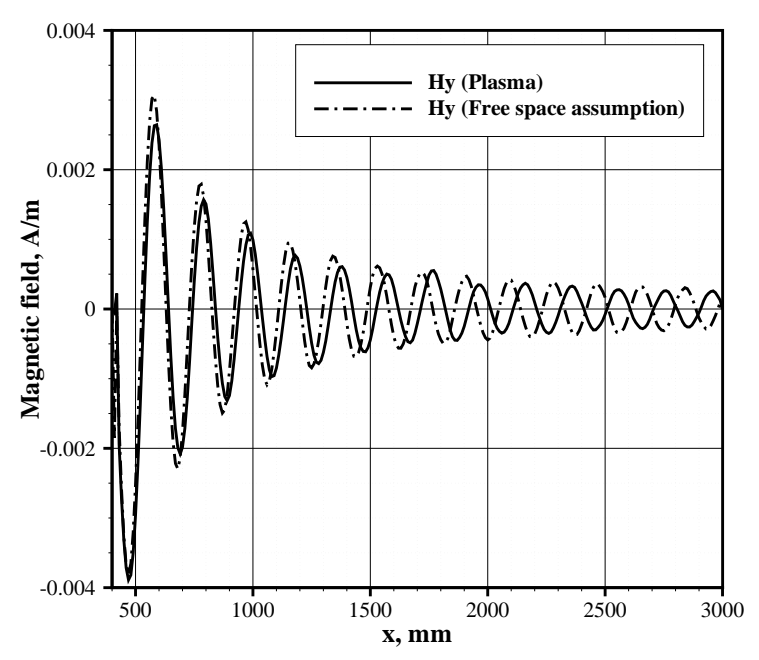

(b) $y$-direction component of electric field $\left(H_{y}\right)$

Figure 12: Axial profiles comparison of electric field and magnetic field along center line ( $y=0 \mathrm{~mm}$ and $z=0 \mathrm{~mm}$ ) of FD2TD computational domain around TITANS at an altitude of $89.3 \mathrm{~km}$.

Figures 13(a), 13(b), 13(c), 13(d), 13(e) and 13(f) show maps of the electric field ratio around TITANS in the $x-y$ plane of the FD2TD computational domain at altitudes of 100.2 $\mathrm{km}, 95.6 \mathrm{~km}, 89.3 \mathrm{~km}, 84.3 \mathrm{~km}, 80.0 \mathrm{~km}$, and $75.6 \mathrm{~km}$, respectively. The electric fields are normalized by the value at the antenna source $\left(|E| / E_{z 0}\right)$. The origin of the argument $(\theta)$ is set at the antenna positions, and the argument is $0^{\circ}$ or $180^{\circ}$ on the $x$-axis. The electric field ratios described in these figures are averaged values over computational results of the FD2DT simulation; the physical model sets of 16 cases and error bars are also shown. Fundamentally, for the present cases, the maps should be evaluated using distantly-positioned electric field magnitudes such as $\sim 100 \mathrm{~m}$. However, because the wave behaves as a plane wave and there exists less ionized gas in the far-field region, electromagnetic wave energy propagates with less decrease and the wave feature changes minimally. From the viewpoint of computational cost and simplicity, in the present study, the electric field at a position $5 \mathrm{~m}$ away from the antenna is selected. Because the antenna is embedded in the aft part of the capsule, the 
electromagnetic wave energy becomes small in the front region of TITANS and large in the rear region $\left(\theta \sim 180^{\circ}\right)$ at each altitude. As with the distributions of the number density of electrons, the dispersion of the electric field among the physical model sets becomes large at high altitude and small at lower altitudes. These tendencies are similar to those of the number density of electrons. The error bar at an altitude of $95.6 \mathrm{~km}$, shown in Fig. 13(b), becomes large because the dispersion among the physical model sets becomes large at this altitude, as discussed in the previous subsection. In the present flow-field simulation, ionized gas in the shock layer in front of TITANS is not generated at altitudes of $100.2 \mathrm{~km}$ and $75.4 \mathrm{~km}$. Thus, electromagnetic waves can propagate without reflection, attenuation, and diffraction by the plasma at these altitudes. On the other hand, at altitudes of $89.3 \mathrm{~km}$ and $84.3 \mathrm{~km}$, wave energies are observed to attenuate almost completely in front of TITANS. This is mainly because the electromagnetic waves can not propagate by the shock layer and plasma wake generated near the torus. On the other hand, the electromagnetic waves propagate in the rear region. A data-relay satellite is expected to always be present behind TITANS during atmospheric reentry and direct communication with the ground station is not considered in the mission. Thus, the loss of data transmission from the front is insignificant compared with that from the back. The view angle of a signal that a data-relay satellite can catch decreases near the maximum heating rate altitude, although no RF blackout phenomena is thought to be observed during flight.

\subsection{Discussion}

The abovementioned results suggest that the RF blackout phenomenon dose not occur during the atmospheric reentry of TITANS and that it is possible to always link with the data-relay satellite. The absence of RF blackout in the wake region is mainly attributable to the low ballistic coefficient and configuration of the inflatable aeroshell. Most electrons and ions are generated in the shock layer in front of the vehicle. On one hand, less chemical reactions including recombination reactions occur in the wake as a result of which the electron density in the wake decreases if the chemical reaction rate in the shock layer decreases. Lower-ballisticcoefficient flight in order to decelerate at high altitudes generally causes the freestream density to be low, and this causes the number of charged particles generated in the shock layer to decrease. From this viewpoint, the large area and low mass of the inflatable vehicle are important features. In addition, the TITANS vehicle forms a large wake region owing to the high flare angle. After plasma flow passes through above the torus, high-temperature gas inflows into the wake with supersonic expansion. Through this process, the plasma becomes cool and rarefied. This could contribute to the decrease in the number density of electrons at the rear and the absence of the RF blackout phenomena during TITANS's flight. In fact, the electromagnetic waves at each altitude simulated here can propagate in rarefied gas region with less attenuation, although reflection and strong attenuation is caused by the plasma wake region near the inflatable torus.

\section{Conclusions}

Numerical simulations of plasma flows around an inflatable reentry vehicle were performed to evaluate the possibility of radio frequency blackout during atmospheric reentry. The flow fields were assumed to be in thermochemical nonequilibrium. To express thermal nonequilibrium accurately, a four-temperature model was applied in the present analysis model. In 
addition, the temperature was separated into translational, rotational, vibrational, and electron temperatures, and detailed internal energy exchange models were utilized. In this study, a mathematical formulation of complicated flow fields in the shock layer was established, and its implementation through an effective numerical procedure was achieved.

The plasma flow simulations were performed for the six cases among altitude $100.2 \mathrm{~km}$ and $75.4 \mathrm{~km}$ according to reentry orbit data based on the calculation results of the equation of motion. Through these computations, the detailed flow-field properties in the shock layer were obtained. In particular, it is important to predict the distributions of the electron number density at each altitude to evaluate the possibility of RF blackout. On the other hand, because a physical model possibly has an inherent discrepancy with the true value, the computational results obtained using the given model combinations could inevitably involve an uncertainty. To try to solve this problem, we performed flow simulations using various model combinations and evaluated the possibility of radio frequency blackout at the rear region of the inflatable vehicle. The number density of electrons was found to be sufficiently small. Additionally, based on the computational results by plasma flow simulation, the electromagnetic wave behaviors were investigated in detail by the FD2TD method. Attenuation and reflection were found to occur in the plasma wake passing near the inflatable torus. However, in the rarefied gas region behind the inflatable vehicle, electromagnetic waves were found to propagate with less attenuation and no reflection. Thus, we concluded that radio frequency blackout phenomena may not occur during the atmospheric reentry of the inflatable vehicle.

\section{References}

[1] K. Yamada, T. Abe, K. Suzuki, N. Honma, M. Koyama, Y. Nagata, D. Abe, Y. Kimura, A. K. Hayashi, D. Akita, and H. Makino. "Deployment and Flight Test of Inflatable Membrane Aeroshell using Large Scientific Balloon". AIAA Paper 2011-2579, 2011.

[2] K. Yamada, T. Abe, K. Suzuki, O. Imamura, D. Akita, M. research, and d group. "Reentry Demonstration Plan of Flare-type Membrane Aeroshell for Atmospheric Entry Vehicle using a Sounding Rocket". AIAA Paper 2011-2521, 2011.

[3] K. Yamada, Y. Nagata, N. Honma, D. Akita, O. Imamura, T. Abe, K. Suzuki, and M. R. group. "Reentry Demonstration Deployable and Flexible Aeroshell for AtmosphericEntry Vehicle using Sounding Rocket". In Proceedings of 63th International Astronautical Congress, AC-12-D2.3.3, Naples, Italy, October 1 - 52012.

[4] K. S. Kunz and R. J. Luebbers. The Finite Difference Time Domain Method for Electromagnetics. CRC Press, Boca Raton, 1993.

[5] M. E. Tauber, J. V. Bowles, and L. Yang. "Use of Atmospheric Braking During Mars Missions". Journal of Spacecraft and Rockets, Vol. 27, No. 5, pp. 514-521, SeptemberOctober 1990.

[6] R. N. Gupta, J. M. Yos, R. A. Thompson, and K.-P. Lee. "A Review of Reaction Rates and Thermodynamic and Transport Properties for an 11-Species Air Model for Chemical and Thermal Nonequilibrium Calculations to 30000 K". NASA RP-1232, Aug. 1990.

[7] C. F. Curtiss and J. O. Hirschfelder. "Transport Properties of Multicomponent Gas Mixture". Journal of Chemical Physics, Vol. 17, No. 6, pp. 550-555, June 1949. 
[8] C. Park. "Assessment of a Two-Temperature Kinetic Model for Dissociating and Weakly Ionizing Nitrogen". Journal of Thermophysics and Heat Transfer, Vol. 2, No. 1, pp. 8-16, Jan.-March 1988.

[9] C. Park. Nonequilibrium Hypersonic Aerothermodynamics. Wiley, New York, 1990.

[10] C. Park. "Rotational Relaxation of $\mathrm{N}_{2}$ Behind a Strong Shock Wave". Journal of Thermophysics and Heat Transfer, Vol. 18, No. 4, pp. 527-533, Oct.-Dec. 2004.

[11] R. C. Millikan and D. R. White. "Systematics of Vibrational Relaxation". The Journal of Chemical Physics, Vol. 39, No. 12, pp. 3209-3213, Dec. 1963.

[12] C. Park. "Problems of Rate Chemistry in the Flight Regimes of Aeroassisted Orbital Transfer Vehicles". AIAA Paper 84-1730, 1984.

[13] J. P. Appleton and K. N. C. Bray. "The Conservation Equations for a Nonequilibrium Plasma". Journal of Fluid Mechanics, Vol. 20, No. 4, pp. 659-672, June 1964.

[14] M. Mitchner and C. H. K. Jr. Partially Ionized Gases. Wiley, New York, 1973.

[15] J.-H. Lee and D. R. White. "Thermal Design of Aeroassisted Orbital Transfer Vehicles". In H. F. Nelson, editor, Basic Governing Equations for the Flight Regimes of Aeroassisted Orbital Transfer Vehicles (Progress in Astronautics and Aeronautics), Vol. 96, pp. 3-53. AIAA, New York, 1985.

[16] M. Nishida and M. Matsumoto. "Thermochemical Nonequilibrium in Rapidly Expanding Flows of High-Temperature Air". Zeitschrift für Naturforcuhung, Teil A: Physik, Physikalische Chemie, Kosmophysik, Vol. 52, pp. 358-368, 1997.

[17] S. S. Lazdinis and S. L. Petrie. "Free Electron and Vibrational Temperature Nonequilibrium in High Temperature Nitrogen". Physics of Fluids, Vol. 17, No. 8, pp. 1539-1546, Aug. 1974.

[18] J.-H. Lee. "Electron-Impact Vibrational Relaxation in High-Temperature Nitrogen". Journal of Thermophysics and Heat Transfer, Vol. 7, No. 3, pp. 399-405, July-Sept. 1993.

[19] P. A. Gnoffo, R. N. Gupta, and J. L. Shinn. "Conservation Equations and Physical Models for Hypersonic Air Flows in Thermal and Chemical Nonequilibrium". NASA TP-2867, Feb. 1989.

[20] Y. Wada and M.-S. Liou. "A Flux Splitting Scheme with High-Resolution and Robustness for Discontinuities". AIAA Paper 94-0083, 1994.

[21] T. R. A. Bussing and E. M. Murman. "Finite-Volume Method for the Calculation of Compressible Chemically Reacting Flows". AIAA Journal, Vol. 26, No. 9, pp. 10701078, 1988.

[22] K. Yamada, Y. Kato, and T. Abe. "Numerical Simulation of Hypersonic Flow around Flare-Type Aeroshell with Torus Frame". In $6^{\text {th }}$ Asia Workshop on Computational Fluid Dynamics, Kashiwa, Japan, March 162009. 
[23] K. Kinefuchi, I. Funaki, and T. Abe. "Frequency-Dependent FDTD Simulation of the Interaction of Microwaves With Rocket-Plume". IEEE Transactions on Antennas and Propagation, Vol. 58, No. 10, pp. 3282-3288, October 2010.

[24] S. A. Cummer. "An Analysis of New and Existing FDTD Methods for Isotropic Cold Plasma and a Method for Improving Their Accuracy". IEEE Transactions on Antennas and Propagation, Vol. 45, No. 3, pp. 392-400, March 1997.

[25] K. S. Yee. "Numerical Solution of Initial Boundary Value Problems Involving Maxwell's Equation Isotropic Media". IEEE Transactions on Antennas and Propagation, Vol. 14, No. 3, pp. 303-307, 1966.

[26] G. Mur. "Absorbing Boundary Conditions for the Finite-Difference Approximation of the Time-Domain Electromagnetic-Field Equations". IEEE Transactions on Electromagnetic Compatibility, Vol. EMC-23, No. 4, pp. 377-382, 1981.

[27] M. Capitelli, C. Gorse, S. Longo, and D. Giordano. "Collision Integrals of HighTemperature Air Species". Journal of Thermophysics and Heat Transfer, Vol. 14, No. 2, pp. 259-268, April-June 2000.

[28] M. Fertig, A. Dohr, and H.-H. Frühauf. "Transport Coefficients for High-Temperature Nonequilibrium Air Flows". Journal of Thermophysics and Heat Transfer, Vol. 15, No. 2, pp. 148-156, April-June 2001.

[29] J. G. Parker. "Rotational and Vibrational Relaxation in Diatomic Gases". The Physics of Fluids, Vol. 2, No. 4, pp. 449-462, July-Aug. 1959.

[30] S. P. Sharma, W. M. Huo, and C. Park. "Rate Parameters for Coupled VibrationDissociation in a Generalized SSH Approximation". Journal of Thermophysics and Heat Transfer, Vol. 6, No. 1, pp. 9-21, January-March 1992. 


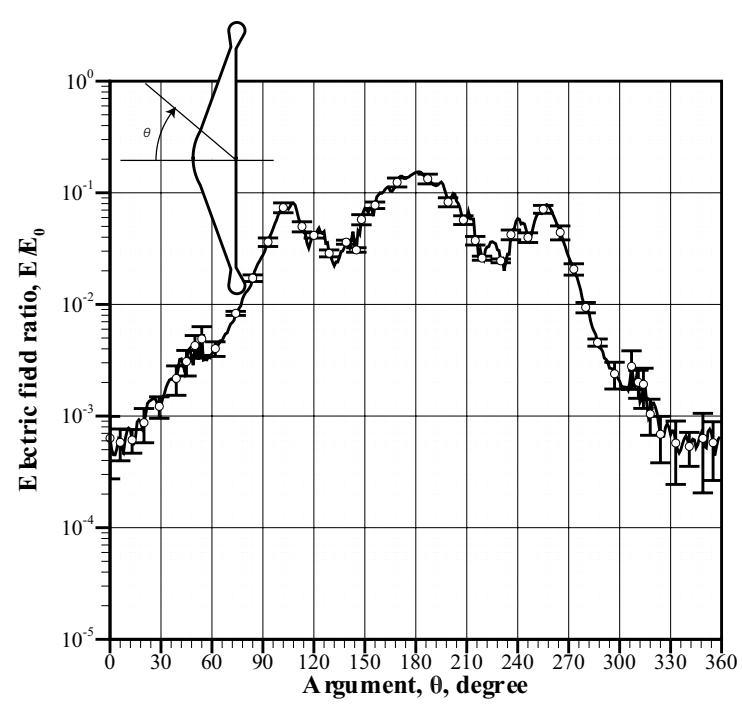

(a) altitude $100.2 \mathrm{~km}$

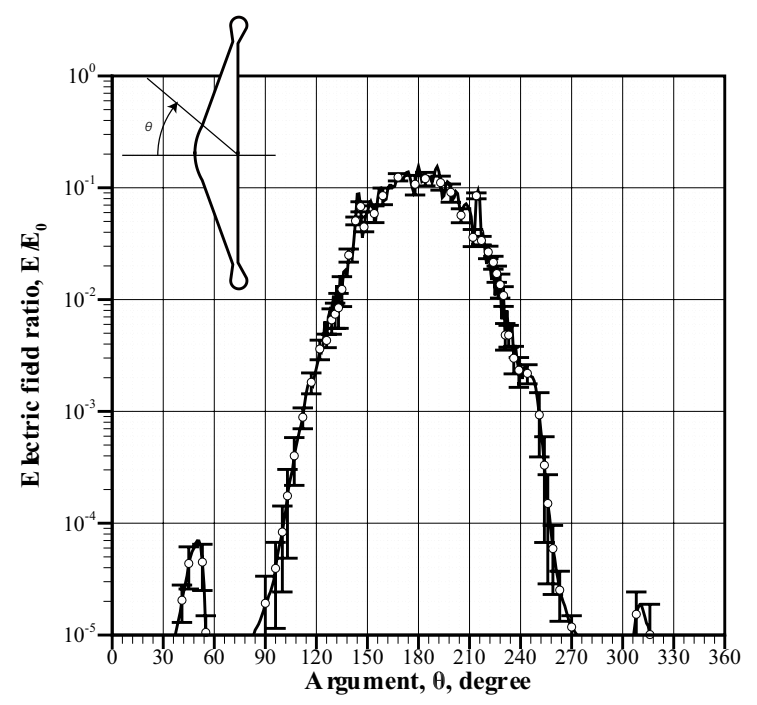

(c) altitude $89.3 \mathrm{~km}$

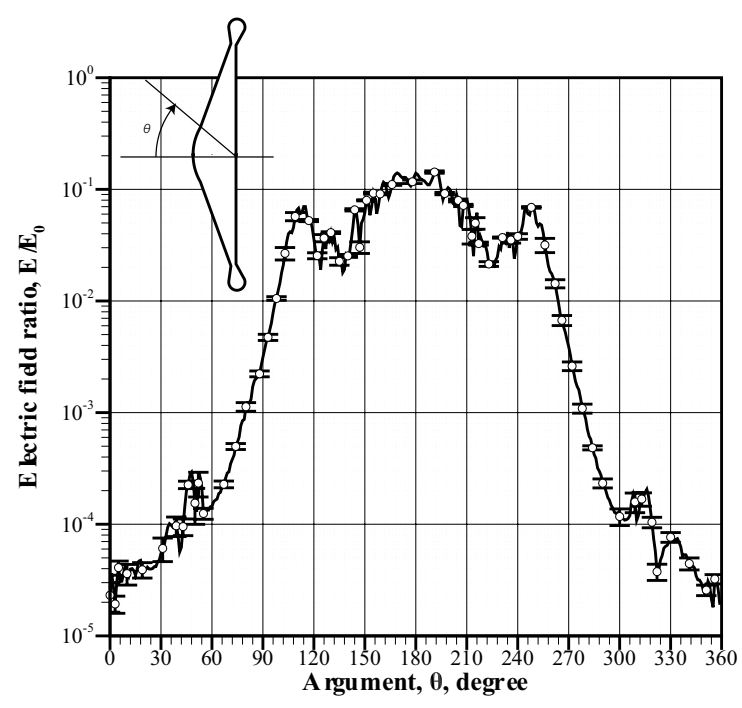

(e) altitude $80.0 \mathrm{~km}$

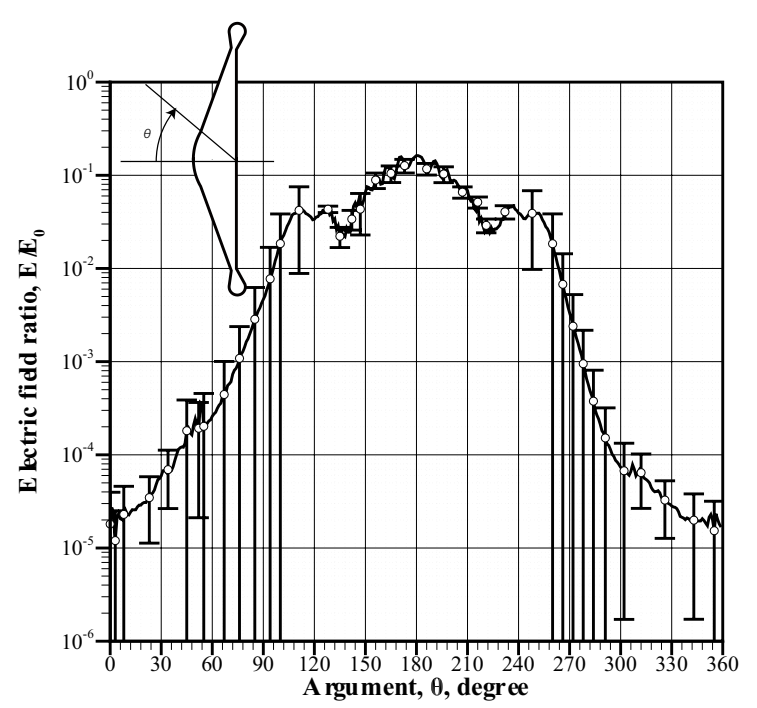

(b) altitude $95.6 \mathrm{~km}$

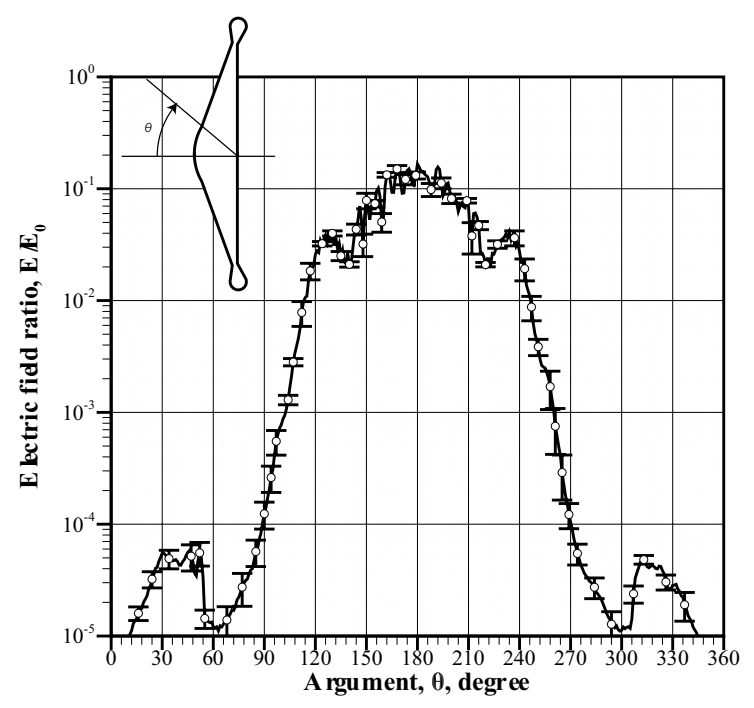

(d) altitude $84.3 \mathrm{~km}$

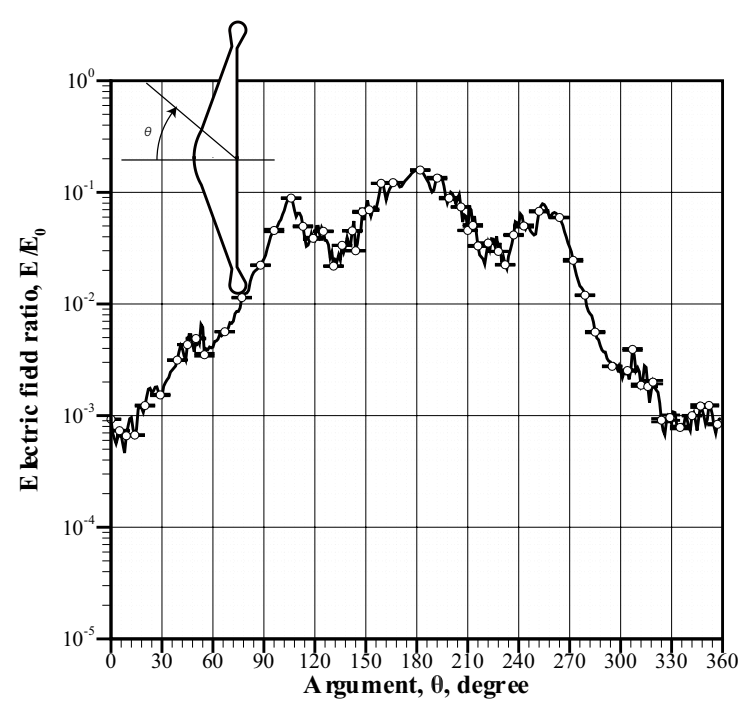

(f) altitude $75.6 \mathrm{~km}$

Figure 13: Electric field maps in $x-y$ plane $(z=0 \mathrm{~mm})$ of FD2TD computational domain around TITANS at each altitude. 